\title{
Laparoscopic Cholecystectomy in a Child Using Slender Forceps
}

\author{
Kazuaki Shibuya, ${ }^{1}$ Hideki Kawamura,,${ }^{1,2 *}$ Munenori Tahara, ${ }^{2}$ Masahiro Takahashi, ${ }^{2}$ Akinobu Taketomi ${ }^{1}$ \\ 1 Department of Gastroenterological Surgery I Hokkaido University Graduate School of Medicine Kita 15, Nishi 7, Kita-ku, Sapporo 060-8638, Japan \\ 2 Department of Surgery, Hokkaido P.W.F.A.C Sapporo Kosei General Hospital Kita 3-Jo Higashi 8-chyome, Chuo-ku, Sapporo 060-0033, Japan
}

\begin{abstract}
Laparoscopic procedures in children are technically demanding because of reduced working space with careful monitoring of pneumoperitoneum pressure. We report a case of laparoscopic cholecystectomy performed in a 9year-old boy using slender forceps which addressed all the above mentioned concerns. This shows a possibility of needlescopic surgery in children.
\end{abstract}

\section{Key words: Pediatric cholelithiasis; Laparoscopy; Needlescopy}

Correspondence*: Hideki Kawamura, Department of Gastroenterological Surgery I Hokkaido University Graduate School of Medicine Kita 15, Nishi 7, Kita-ku, Sapporo 060-8638, Japan

E-mail: h.kawamura@med.hokudai.ac.jp @ @ 2017, Shibuya et al.

Submitted: 12-02-2017

Accepted: 21-03-2017

Conflict of Interest: None

Source of Support: Nil

This is an open-access article distributed under the terms of the Creative Commons Attribution License, which permits unrestricted use, distribution, and reproduction in any medium, provided the original work is properly cited.

\section{INTRODUCTION}

Cholelithiasis in children is not an uncommon condition. Laparoscopic cholecystectomy is a preferred mode of treatment. Laparoscopy in children is challenging as surgeons have to work in a restricted space with low pressure pneumoperitoneum [1]. Single port laparoscopy using slender forceps is increasingly practiced nowadays [2]. In pediatric laparoscopic surgery, slender forceps are useful in performing appendectomy, colectomy, and inguinal hernia repair [3]. Herein we describe surgical technique of laparoscopic cholecystectomy using slender forceps.

\section{CASE REPORT}

A 9-year-old boy (height: $131.3 \mathrm{~cm}$, weight: $22.7 \mathrm{~kg}$ ) with the diagnosis of cholelithiasis was booked for laparoscopic cholecystectomy.
After preoperative optimization, a small abdominal incision for insertion of the camera port (5-mm) was made in umbilicus. The pressure of pneumoperitoneum was kept at $6 \mathrm{mmHg}$, which is lower than that for an adult (8 - $10 \mathrm{mmHg}$ ). To use ultrasonically activated device (USAD), a 5-mm port was inserted below the xiphoid process, but to reduce the interference of the forceps within the restricted working space and for aesthetic considerations, two 2-mm diameter grasper forceps (BJ needle; NITI-ON, Chiba, Japan) were inserted in the right hypochondrium for the surgeon's left hand and the assistant. Cystic artery was coagulated with USAD. Cystic duct was double ligated using 4-0 absorbable sutures. Duration of surgery was $45 \mathrm{~min}$, and there was minimal blood loss. A 9-mm black stone was found within the gallbladder. Calculus analysis indicated that its composition was $36 \%$ calcium carbonate, $33 \%$ calcium phosphate, and $31 \%$ calcium bilirubinate. The patient was discharged on the fourth day after surgery without any complication. 


\section{DISCUSSION}

Laparoscopic cholecystectomy in children requires several adjustments in technique. In the present case, because of the restricted space we used slender forceps. We practice needlescopic surgery for the colon related operations as well as single port laparoscopic cholecystectomy at our institute. We applied our knowledge and skill for needlescopic cholecystectomy for the first time in our hospital. In our case, abdominal pressure was maintained at $6 \mathrm{mmHg}$ and no effect on respiratory dynamics or hemodynamics was observed, and the surgery could be performed while maintaining a satisfactory operative field. In conclusion, slender forceps can be used to perform cholecystectomy safely thus promoting needle- scopic surgery which further have benefits of barely visible scars and negligible postoperative pain compared to conventional laparoscopy.

\section{REFERENCES}

1. Okada T, Sasaki B, Kataoka A. Topics in surgical treatment: Laparoscopic surgery in children. Hokkaido J Surg. 2009;54:2-7.

2. Bibza J, Sykora L, Cingel V, Duchaj B, Babala J, Kralik R, et al. Laparoscopic cholecystectomy in children. Bratisl Lek Listy. 2013;114:230-1.

3. Deepak J, Agarwal P, Bagdi RK, Balagopal S, Madhu R, Balamourougane P. Pediatric cholelithiasis and laparoscopic management: A review of twenty two cases. J Minim Access Surg. 2009;5:93-6. 